13. Wijewantha HS, Sellahewa KH. Hump nosed viper bite in Sri Lanka - descriptive observational study of 1543 cases. Asian Pac J Trop Med. 2010;3(11):902-5.

\section{A Comparison of 4 Methods to Make Improvised Suture Needles}

\section{To the Editor:}

Lacerations are a common injury in the wilderness. A 2008 study calculated that lacerations comprised approximately $15 \%$ of wilderness injuries assessed in emergency departments in the United States. ${ }^{1}$ The Wilderness Medical Society (WMS) clinical practice guidelines for basic wound management endorse the primary closure of most wounds in the wilderness. ${ }^{2}$ Commercial suture needles may be unavailable for laceration repair in the wilderness, so methods to improvise suture needles have been developed. These techniques are described in textbooks ${ }^{3,4}$ and were taught in a workshop during the WMS 2020 winter conference. After attending this workshop, I reviewed the primary literature looking for evidence on the effectiveness of these techniques. Studies comparing improvised to commercial sutures have been performed and show that sutures made with fishing line promote wound healing as effectively as commercial sutures. ${ }^{5,6}$ I found no studies, however, that assessed whether improvisation methods are effective at producing a functional suture needle. I therefore decided to perform a small experiment to compare the functionality of different methods of improvising suture needles.

The materials used to construct the improvised suture needles were hypodermic needles and fishing line. I began by trialing $25 \mathrm{~mm}$ ( 1 in) 25 ga hypodermic needles and then repeated some of the trials with 22 ga needles (Becton Dickinson). The fishing line used was $0.215 \mathrm{~mm}$ (0.008 in) monofilament fishing line with a weight rating of $2.7 \mathrm{~kg}$ (6.8 lb; Rapala VMC Corp).

I assessed 4 methods of making improvised suture needles: the swaged method, the double backed method, and the looped method with 2 different knots. In the swaged suture, material was drawn through the hypodermic needle, and a multitool was used to crimp the bottom $20 \mathrm{~mm}$ of the needle to anchor the suture material in place (Figure 1A). Both the needle hub and shaft were crimped to maximize the anchoring effect because I observed many anchor failures when only crimping the needle hub during previous trials. In the double backed suture, material was drawn through the hypodermic needle, leaving at minimum $5 \mathrm{~cm}$ of suture material running out of the beveled end of the needle. The beveled end of the suture material was then folded backward toward the hub end of the needle. The suture material was not affixed to the needle (Figure 1B). In the looped suture, material was drawn through the hypodermic needle, leaving approximately $10 \mathrm{~cm}$ of suture material running out of the beveled end. A loop was then formed by tying the suture material back onto itself with the needle
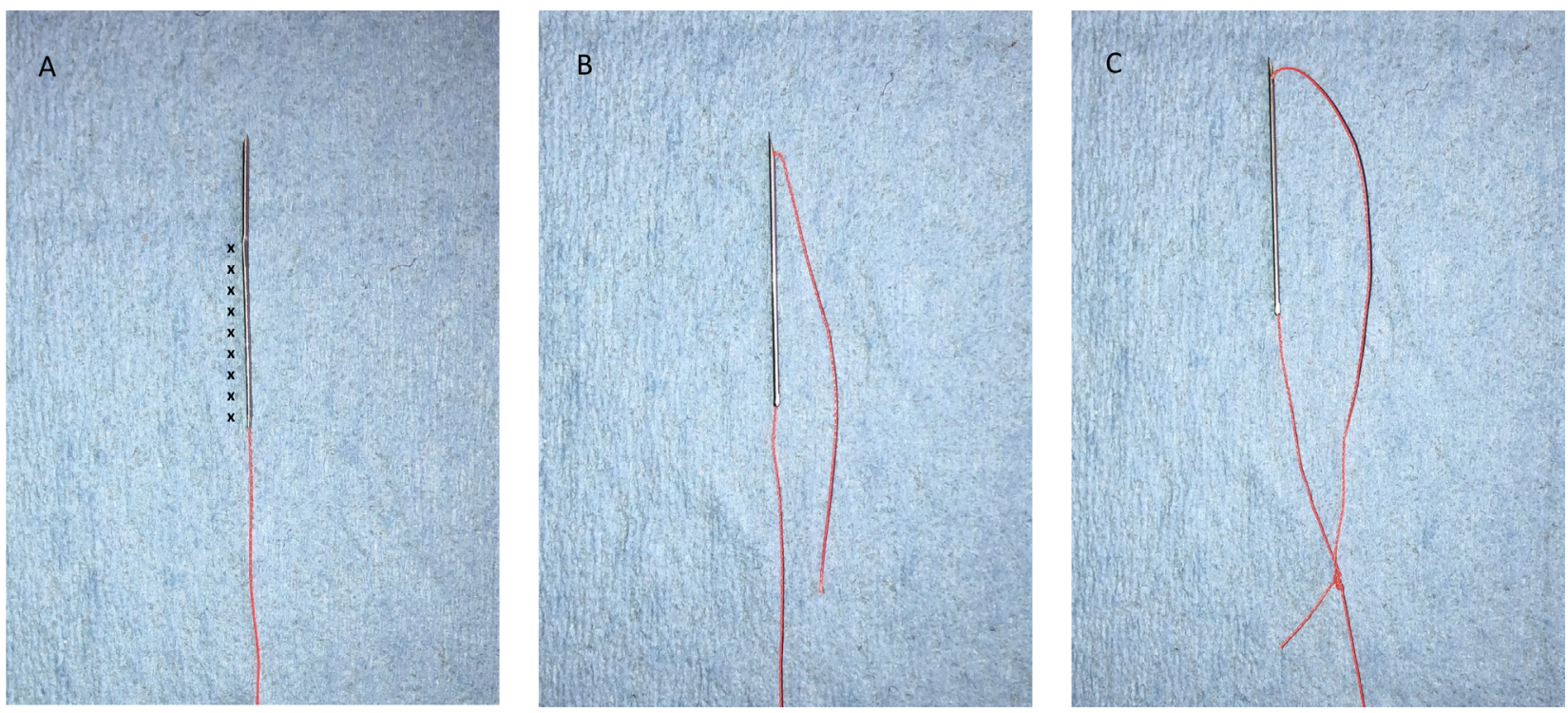

Figure 1. Examples of improvised suture needles. (A) A suture needle made with the swaged technique, where $x$ indicates the portion of the needle that has been crimped. (B) A suture needle made with the double-backed method. (C) A suture needle made with the looped method, using a nonslip loop knot. Note that 18 ga hypodermic needles and $0.23 \mathrm{~mm}$ orange fishing line have been used in these photographs so that the materials are better visualized. 


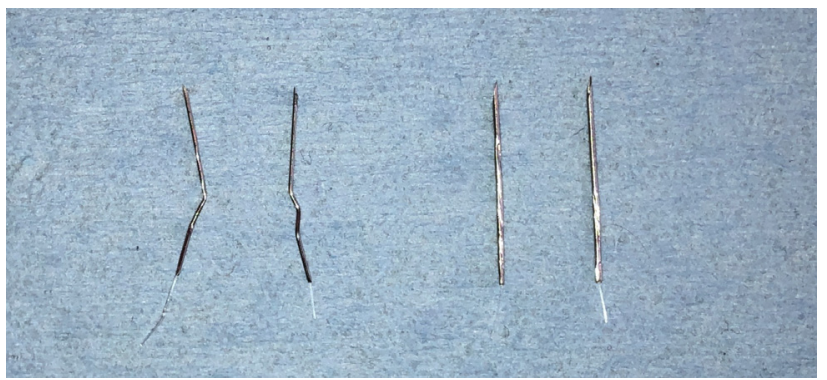

Figure 2. Swaged suture needles after completion of 10 simple interrupted sutures. The 2 suture needles on the left were made with $25 \mathrm{ga}$ hypodermic needles and were significantly misshaped after use. The 2 suture needles on the right were made with 22 ga hypodermic needles and retained their shape.

incorporated into the loop. The 2 knots trialed for the loop method were an overhand on a bight and a nonslip loop knot (or Kreh knot) (Figure 1C).

To test the functionality of these improvised suture needles, I used the needles to make simple interrupted sutures in a commercial silicone suture training pad. In each trial, a single needle was used until 10 simple interrupted sutures were completed or the needle failed, whichever occurred first. Failure of the needle meant either the suture material came free of the needle or the shaft of the needle broke. Ten trials were completed of each of the 4 suture construction methods using suture needles built with 25 ga hypodermic needles. An additional 10 trials were performed for the swaged and double backed methods using suture needles built with 22 ga hypodermic needles. A total of 60 trials were performed.

When 25 ga hypodermic needles were used, the double backed method was the most successful improvisation method, experiencing no failures. The swaged method experienced 2 failures, both caused by a break of the needle shaft within the crimped area. The looped method experienced a high frequency of failure $(>50 \%)$ regardless of the knot used. These all involved the knot getting caught in the silicone material, followed by a break in the fishing line somewhere within the loop. Across all 4 methods, I noted that the 25 ga needle was significantly misshaped and difficult to use once the needle had been used to complete 10 sutures (Figure 2). When 22 ga hypodermic needles were used, the double backed method was the most successful method, again experiencing no failures. The swaged method experienced 3 failures, all due to the suture material breaking free from the needle. In contrast to the 25 ga needles, the
22 ga needles retained their shape and functionality well after being used to complete 10 sutures (Figure 2).

This small experiment provides some evidence supporting improvisation methods to produce functional suture needles in an in vitro model. The double backed method stands out as the only technique that performed without failure. Of the methods assessed here, the double backed method was also the simplest to assemble: It does not require access to a multitool, and because it does not require the ability to tie knots, it may be easier to assemble sutures using this method while wearing insulated gloves or with reduced fine motor dexterity due to cold exposure.

I hope that these findings may be of interest to the wilderness medicine clinician. Further studies could look at validating these findings across multiple providers, in outdoor settings, using tissue models, and trialing alternative materials.

\section{David Jerome, MD, MSc, FAWM Department of Family Medicine \\ University of Alberta \\ Division of Clinical Sciences \\ Northern Ontario School of Medicine \\ Edmonton, $A B$}

\section{References}

1. Flores AH, Haileyesus T, Greenspan AI. National estimates of outdoor recreational injuries treated in emergency departments, United States, 2004-2005. Wilderness Environ Med. 2008;19(2):91-8.

2. Quinn RH, Wedmore I, Johnson EL, Islas AA, Anglim A, Zafren K, et al. Wilderness Medical Society practice guidelines for basic wound management in the austere environment: 2014 update. Wilderness Environ Med. 2014;25(4 Suppl):S118-33.

3. Iserson VK, Macias DJ. Improvised medicine in the wilderness. In: Auerbach PS, ed. Wilderness Medicine. 7th ed. Philadelphia, PA: Elsevier; 2017:1046-80.

4. Iserson VK. Surgical equipment. In: Improvised Medicine: Providing Care in Extreme Environments. 2nd ed. New York, NY: McGraw-Hill Education; 2016:349-62.

5. Freudenberg S, Mkony C, Wilhelm T, Nyawawa T, Kuhn C, Grobholz R, et al. Atraumatic intracutaneous skin closure with self-made fishing line suture compared to commercial thread. East Afr Med J. 2004;81(7):348-52.

6. Freudenberg S, Nyonde M, Mkony C, Bay F, Wilhelm T, Post S. Fishing line suture: cost-saving alternative for atraumatic intracutaneous skin closure - randomized clinical trial in Rwanda. World J Surg. 2004;28(4):421-4. 